\title{
Regulation of noise in the expression of a single gene
}

\author{
Ertugrul M. Ozbudak ${ }^{1}$, Mukund Thattai ${ }^{1}$, Iren Kurtser ${ }^{2}$, Alan D. Grossman ${ }^{2}$ \& Alexander van Oudenaarden ${ }^{1}$
}

Published online: 22 April 2002, DOI: 10.1038/ng869

Stochastic mechanisms are ubiquitous in biological systems. Biochemical reactions that involve small numbers of molecules are intrinsically noisy, being dominated by large concentration fluctuations ${ }^{1-3}$. This intrinsic noise has been implicated in the random lysis/lysogeny decision of bacteriophage- $\lambda^{4}$, in the loss of synchrony of circadian clocks ${ }^{5,6}$ and in the decrease of precision of cell signals ${ }^{7}$. We sought to quantitatively investigate the extent to which the occurrence of molecular fluctuations within single cells (biochemical noise) could explain the variation of gene expression levels between cells in a genetically identical population (phenotypic noise). We have isolated the biochemical contribution to phenotypic noise from that of other noise sources by carrying out a series of differential measurements. We varied independently the rates of transcription and translation of a single fluorescent reporter gene in the chromosome of Bacillus subtilis, and we quantitatively measured the resulting changes in the phenotypic noise characteristics. We report that of these two parameters, increased translational efficiency is the predominant source of increased phenotypic noise. This effect is consistent with a stochastic model of gene expression in which proteins are produced in random and sharp bursts. Our results thus provide the first direct experimental evidence of the biochemical origin of phenotypic noise, demonstrating that the leve of phenotypic variation in an isogenic population can be regulated by genetic parameters.

We selected as our reporter system a single-copy chromosomal gene with an inducible promoter. As an estimated $50-80 \%$ of bacterial genes are transcriptionally regulated ${ }^{8}$, this system typifies the majority of naturally occurring genes, allowing our results to be extended to natural systems. We incorporated a single copy of our reporter, the green fluorescent protein gene $(g f p)$ into the chromosome of $B$. subtilis. We chose to integrate $g f p$ into the chromosome itself, rather than in the form of plasmids, as variation in plasmid copy number ${ }^{9,10}$ can act as an additional and unwanted source of noise. Transcriptional efficiency was regulated by using an isopropyl- $\beta$-D-thiogalactopyranoside (IPTG)-inducible promoter, $\mathrm{P}_{\text {spac }}$, upstream of $g f p$, and varying the concentration of IPTG in the growth medium. Translational

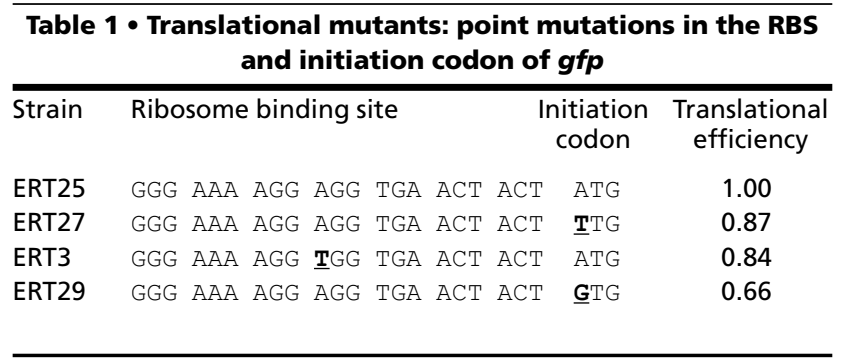

efficiency was regulated by constructing a series of B. subtilis strains (Table 1) that contained point mutations in the ribosome binding site (RBS) and initiation codon of $g f p^{11}$. The use of two different strategies to regulate transcriptional and translational processes introduces a potential bias in the relative contributions of these processes to biochemical noise. As a control, we constructed four additional strains (Table 2) whose transcription rates were altered by mutations in the promoter region of the reporter gene. As described below, both strategies of transcriptional regulation produced similar results.

We measured expression of green fluorescent protein (GFP) for single cells in a bacterial population using flow cytometry. Variation in GFP expression from cell to cell (phenotypic noise) is seen in a histogram (Fig. 1a) showing the protein expression levels $(p)$ measured during a typical experiment. The histogram is characterized by a mean value $\langle p\rangle$ and a standard deviation $\sigma_{p}$. The phenotypic noise strength, defined as the quantity $\sigma_{p}^{2}\langle\langle p\rangle$ (variance/mean), is sensitive to the biochemical sources of stochasticity that we wished to study and is therefore the unit in which we report our results. We measured phenotypic noise strength for the four different translational strains as we varied IPTG concentration between $30 \mu \mathrm{M}$ (near-basal transcription) and $1 \mathrm{mM}$ (full operon induction). For example, Fig. $1 b$ shows flow cytometer results for the four strains at full induction, whereas Fig. $1 c$ shows the results from a series of flow cytometer experiments conducted on a single strain (ERT3) as IPTG concentration was varied. A summary of all of our experimental results (Fig. $2 a$ ) shows the measured noise strength as a simultaneous function of both transcriptional efficiency (varying [IPTG] in the growth medium) and translational efficiency (using different strains with mutations in the RBS and initiation codon). As the addition of IPTG and mutations in the $g f p$ RBS are not expected to affect normal cellular processes, all contributions to phenotypic noise remained unchanged throughout our experiment, except fluctuations in rates of transcription and translation. The response of phenotypic noise strength to a change in either translational efficiency (Fig. $2 b$ ) or transcriptional efficiency (Fig. $2 c$ ) indicates the isolated contribution of that parameter to the phenotypic noise.

\begin{tabular}{|c|c|c|}
\hline \multicolumn{3}{|c|}{$\begin{array}{l}\text { Table } 2 \cdot \text { Transcriptional mutants: point mutations } \\
\text { in the } P_{\text {spac }} \text { promoter }\end{array}$} \\
\hline Strain & $\begin{array}{l}-10 \text { regulatory region } \\
-10+1\end{array}$ & Transcriptional efficiency \\
\hline ERT57 & CAT AAT GTG TGT AAT & 6.63 \\
\hline ERT25 & CAT AAT GTG TGG AAT & 1.00 \\
\hline ERT53 & CAT AAT GTG TGC AAT & 0.79 \\
\hline ERT51 & CAT AAT GTG TGE AAT & 0.76 \\
\hline ERT55 & CAT AAT GTG TAA AAT & 0.76 \\
\hline
\end{tabular}

${ }^{1}$ Department of Physics and ${ }^{2}$ Department of Biology, Massachusetts Institute of Technology, Cambridge, Massachusetts 02139, USA. Correspondence should be addressed to A.v.O. (e-mail: avano@mit.edu). 


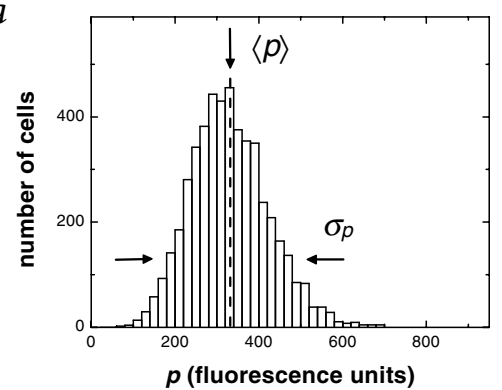

$b$

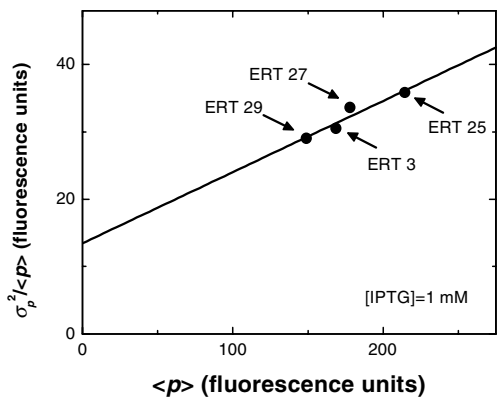

$c$

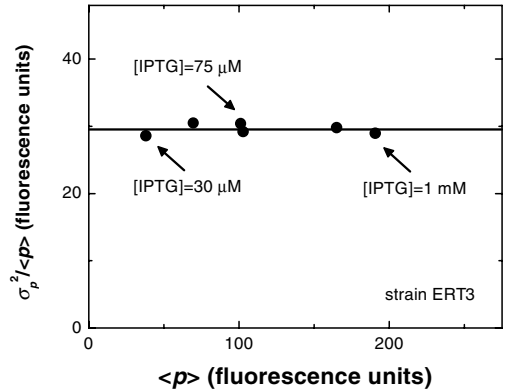

Fig. 1 Phenotypic noise in a genetically identical bacterial population. $a$, Histogram showing the result of a typical experiment in which the expression level of a fluorescent reporter protein is measured in a population of isogenic bacterial cells. Traditional population-averaged measurements would summarize the entire histogram by its mean value $\langle p\rangle$; however, our single-cell measurements show that the expression level varies from cell to cell, with a standard deviation $\sigma_{p}$. The phenotypic noise strength, defined as the quantity $\sigma_{p}{ }^{2} /\langle p\rangle$, is a measure of the spread of expression levels in a population. The relative standard deviation $\sigma_{p} /\langle p\rangle$, although a more common measure of phenotypic noise, obscures its essential behavior. For instance, the relative standard deviation for a Poisson distribution is $\sigma_{P} /\langle p\rangle=1 /\langle p\rangle^{1 / 2}$, which decreases as the mean increases; but the noise strength for this distribution, $\sigma_{P}^{2} /\langle p\rangle=1$, is independent of the mean. In general, the noise strength circumvents the trivial effect of decreased noise with increased mean, and measures deviations from Poisson behavior. $\boldsymbol{b}$, Phenotypic noise strength for the four different translational mutants at fixed inducer concentration. Noise strength is clearly dependent on translational efficiency. c, Phenotypic noise strength for one strain (ERT3) as inducer concentration is varied. The transcriptional efficiency does not significantly affect noise strength.

We find that the phenotypic noise strength shows a strong positive correlation with translational efficiency (Fig. $2 b$, slope $=21.8$, in contrast to the weak positive correlation observed for transcriptional efficiency (Fig. 2c, slope=6.5). Switching from the ERT27 strain to the ERT25 strain (an increase in translational efficiency of about 15\%; Table 1) increases the noise strength from 32 to 35 units; the same effect is achieved only upon doubling transcriptional efficiency (a $100 \%$ increase) from the half-induction to the full-induction level. Experiments involving the control strains, in which transcription rates were altered by mutation rather than by operon induction, supported the weak correlation between noise strength and transcriptional efficiency (Fig. $2 c$ inset, slope $=7.3$ ). The differential nature of our measurements (investigating changes rather than absolute values) makes our results independent of the specific properties of the reporter protein, such as gene locus or folding characteristics. This suggests that increased translational efficiency will strongly increase the variation in the expression of any naturally occurring gene.

A stochastic model for the expression of a single gene (Fig. $3 a$ ) predicts that the noise strength $\left(\sigma_{p}^{2} /\langle p\rangle\right)$ is greater than Poissonian $\left(\sigma_{p}^{2} /\langle p\rangle=1\right)$ and is simply an increasing function of translational efficiency ${ }^{12}$ :

$$
\langle p\rangle=k_{R} b / \gamma_{P}, \quad \frac{\sigma_{p}^{2}}{\langle p\rangle} \cong 1+b .
$$

Here, $b=k_{P} / \gamma_{R}$ is the average number of proteins synthesized per mRNA transcript; these proteins are injected into the cytoplasm in sharp bursts (Fig. 3b). The measured asymmetry between the noise contributions of transcriptional and translational parameters is consistent with this prediction and provides evidence of

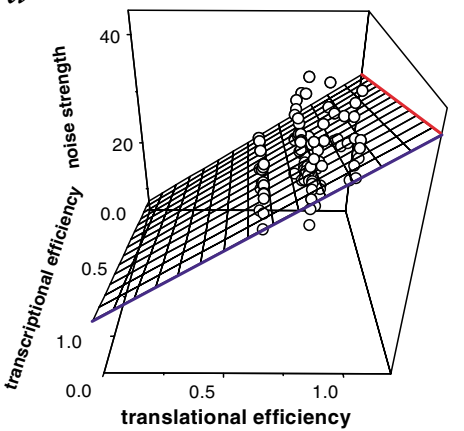

$b$

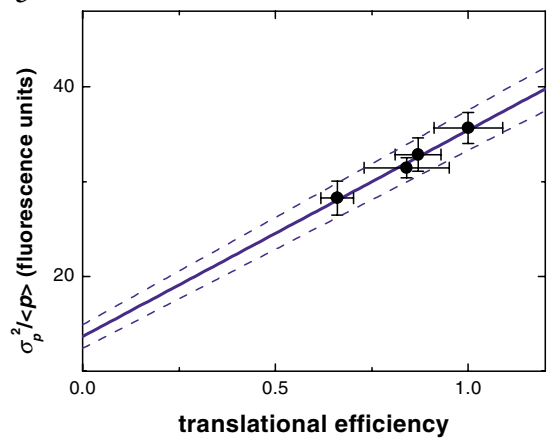

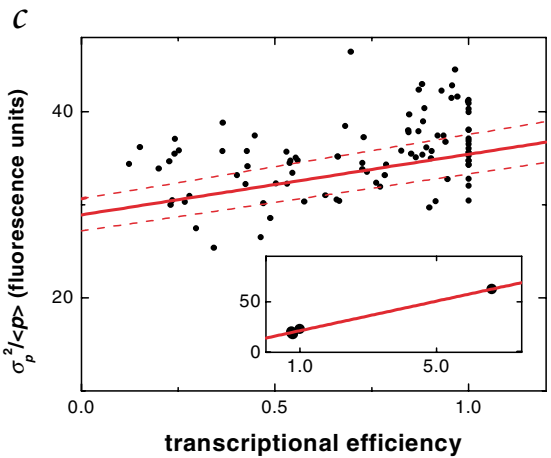

Fig. 2 Biochemical contribution to phenotypic noise. a, Complete experimental data. Each data point is the summarized result of an entire histogram corresponding to a flow cytometer run of a population of typically $10^{4}-10^{5}$ cells. The phenotypic noise strength of the population ( $z$, in arbitrary fluorescence units) is plotted as a function of transcriptional efficiency ( $x$, depending on the IPTG concentration) and translational efficiency ( $y$, depending on the translational mutant used). Transcriptional and translational efficiencies are normalized to those of the wildtype ERT25 strain, allowing these parameters to be directly compared. These data are fitted to a plane of the form $z=a_{0}+a_{x} x+a_{y} y$ using a least-square routine, giving $a_{0}=7.1 \pm 0.9, a_{x}=6.5 \pm 0.4, a_{y}=21.8 \pm 0.9$. The ratio $a_{y} / a_{x}=3.4$ gives the relative effect of translational versus transcriptional efficiency on phenotypic noise strength. $\boldsymbol{b}, \boldsymbol{c}$, For clarity, the three-dimensional data are projected parallel to the fit plane onto the boundary planes $x=1(b)$, noise strength as a function of translation, and $y=1(c)$, noise strength as a function of transcription. The intersection of the fit plane with each boundary plane is shown as a solid line; dotted lines indicate an interval of 1 s.d. Data in $b$ are summarized separately for each translational mutant (dark circles with error bars that represent $95 \%$ c.i.). Inset in c shows results of control experiments conducted on transcriptional mutants at full induction. Three strains (ERT51, ERT53 and ERT55) are very similar, both in transcriptional efficiency and in noise strength, suggesting that biochemical noise is determined by the actual transcription rate rather than by the specific method used to achieve it. The strain ERT57 shows a highly amplified transcriptional efficiency, allowing reliable estimation of correlations. Data are summarized separately for each transcriptional mutant. A linear fit through these points gives a slope $a_{x}^{\prime}=7.3 \pm 0.3$, which is consistent with the slope $a_{x}=6.5 \pm 0.4$ obtained from $a$. 
Fig. 3 The burst size effect. a, Modeling single-gene expression. mRNA mole cules are transcribed at rate $k_{R}$ from the template DNA strand. Proteins are translated at a rate $k_{p}$ from each mRNA molecule. Proteins and mRNA degrade at rates $\gamma_{P}$ and $\gamma_{R}$, respectively. Degradation into constituents is denoted by a slashed circle. $\boldsymbol{b}$, Typically, mRNA is unstable when compared with the protein product of a gene. During its brief lifetime, however, an mRNA molecule can inject a large burst of proteins into the cytoplasm. A Monte Carlo timecourse over a 30 min time interval shows bursts of protein creation of average size $b=k_{p} / \gamma_{R}$ occurring at average rate $k_{R}$. The magnitudes of these parameters are indicated on the figure by bars. The timecourse in $b$ is a magnified section of $c$. $\boldsymbol{c}, \boldsymbol{d}$, Monte Carlo simulations of typical timecourses for protein number. Deterministic timecourses are indicated as solid lines; the corresponding population histogram is shown to the right of each timecourse. The following examples both achieve the same mean protein concentration, but with different noise characteristics. In both cases, $\gamma_{R}=0.1 \mathrm{~s}^{-1}$ and $\gamma_{P}=0.002 \mathrm{~s}^{-1}$; the burst size $b$ is varied to obtain different noise strengths, whereas the transcript initiation rate $k_{R}$ is chosen to fix the mean protein number at 50. A gene with low transcription but high translation rates $\left(c ; k_{R}=0.01 \mathrm{~s}^{-1}, b=10\right)$ produces bursts that are large, variable and infrequent, resulting in strong fluctuations. Conversely, a gene with high transcription and low translation rates $\left(d, k_{R}=0.1 \mathrm{~s}^{-1}, b=1\right)$ produces bursts that are small and frequent, causing only weak fluctuations in protein concentration and producing a smaller phenotypic variation in the population. Regulation of a two-step process, that of transcription followed by translation, can therefore be used to independently adjust the mean protein concentration and the level of phenotypic noise in a bacterial population.

the biochemical origin of phenotypic variability (Fig. $3 c, d$ ). Phenotypic noise in a population is therefore indicative of protein concentration fluctuations over time in single cells.

Cell-to-cell variation in gene expression and flucuations over time in single cells have broad implications. Noise is often harmful, as it garbles cell signals, corrupts circadian clocks ${ }^{6}$ and disrupts the fine-tuned process of development. Cell signaling pathways ${ }^{13}$ and developmental switches ${ }^{14}$ have evolved so as to minimize the disruptive effect of such fluctuations, in ways that are only now beginning to be understood. Recently, Becskei and Serrano reported that variation in gene expression could be reduced by autoregulation ${ }^{15}$. We have shown that phenotypic variation can be controlled by genetic parameters: low translation rates will lead to reduced fluctuations in protein concentration. Because our control parameters are general, our results should be generally applicable. We suggest that several inefficiently translated regulatory genes (Table 3 ) have been naturally selected for their low-noise characteristics, even though efficient translation is energetically favorable ${ }^{16}$. For example, the cya gene of Escherichia coli, whose downstream product cyclic AMP (cAMP) is involved in several cellular regulatory processes, has a low translation rate. The unusual and inefficient RBS of $c y a$ is conserved across a variety of Gram-negative bacteria ${ }^{17}$, perhaps because it suppresses harmful fluctuations in cAMP levels that could have highly pleiotropic effects, including cell death ${ }^{18}$. In some circumstances, noise can be highly desirable: an organism could use high translation rates and large concentration fluctuations as a means of creating nongenetic individuality in a population $^{19,20}$. This is seen with the $c I$ gene of $\lambda$-phage ${ }^{4,21}$ : upon infection of a host cell, the $c I$ mRNA is transcribed with an efficient RBS upstream of the initiation codon, thus creating a high-

Table 3 • Examples of genes inefficiently translated in Escherichia coli

\begin{tabular}{ll}
\hline Gene & Function of gene product \\
$c l$ & regulator of bacteriophage- $\lambda \mathrm{O}_{\mathrm{R}}$ operator $^{21}$ \\
$c y a$ & synthesis of cAMP17 \\
malT & regulator of maltose regulon ${ }^{16}$ \\
nagC & regulator of nag regulon \\
$t e t R$ & regulator of tetracycline resistance \\
$\operatorname{trp} R$ & repressor of trp, trp $R$ and aroH operons ${ }^{28}$
\end{tabular}

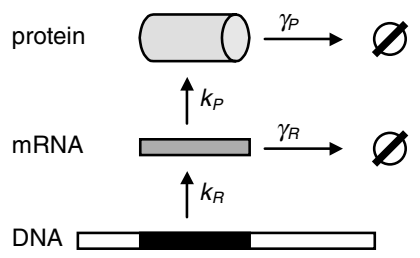

$b$

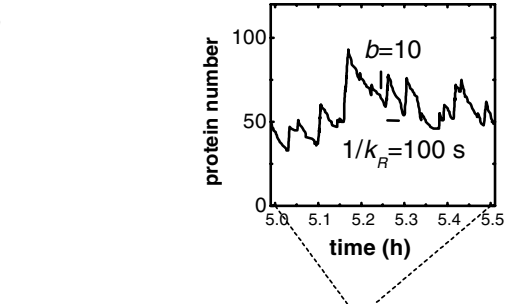

c

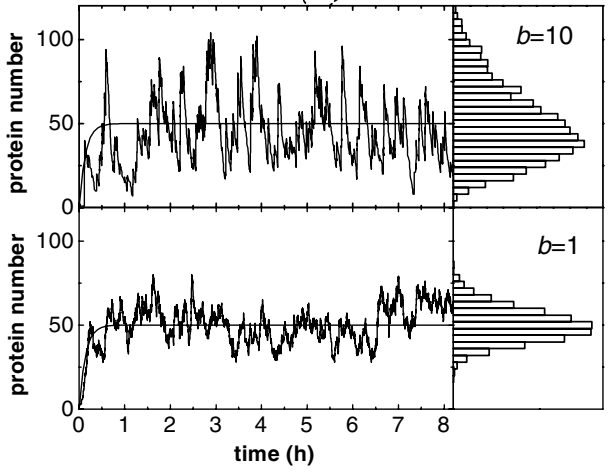

noise state; however, the lysogenic phenotype, once established, is maintained in a low-noise state (since transcription then begins at the initiation codon itself, producing inefficiently translated $\mathrm{mRNA}^{4}$ ). Our experimental approach of creating lownoise genes through the use of inefficient RBSs mirrors the structure of these natural systems. The technique of translational noise control can be applied in the fast-growing field of artificial genetic networks ${ }^{22,23}$. The current capabilities of artificially engineered circuits such as genetic toggle switches ${ }^{24}$ or ring oscillators $^{5}$ are limited by intrinsic noise. New methods of noise reduction will allow these circuits to mimic the robust behavior of natural biological systems and will enable their practical application in areas such as biocomputation or the construction of genetic biosensors.

\section{Methods}

Strains, growth conditions and media. We placed the gene gfpmut 2 under the control of the $\mathrm{P}_{\text {spac }}$ promoter and introduced mutations in the ribosome binding site, initiation codon and promoter region of gfpmut 2 by PCR. Mutations were verified by sequencing; spontaneous mutation frequencies were negligible over the timecourse of our experiments. We digested the PCR products and ligated them into the amyE integration vector pDR67, which contains a single copy of lacI downstream of the constitutive promoter $\mathrm{P}_{\text {pen. }}$. We amplified the resulting recombinant plasmid in the E. coli AG1111 strain and inserted it into the chromosome of the B. subtilis JH642 strain by double-crossover at the amyE locus. (Cells of E. coli and B. subtilis were made competent and transformed according to standard procedures.) The resulting $B$. subtilis strain contained a single copy of gfpmut2 under the $\mathrm{P}_{\text {spac }}$ promoter and a single copy of lacI under the constitutive $\mathrm{P}_{\text {pen }}$ promoter. The $\mathrm{P}_{\text {spac }}$ promoter includes a binding site for Lac repressor, the product of the lacI gene; $\mathrm{P}_{\text {spac }}$ is externally inducible by IPTG, which binds to and inhibits the repressor function of LacI. The concentration of IPTG in the growth medium therefore determines the transcriptional efficiency of $g f p m u t 2$. Addition of IPTG is not expected to affect native operon expression in B. subtilis. 
We grew cells overnight in Luria Bertani (LB) broth at $37^{\circ} \mathrm{C}$, diluted these cultures and induced them with varying amounts of IPTG for at least $5 \mathrm{~h}$ at $37^{\circ} \mathrm{C}$. We grew non-induced strains to determine the amount of background fluorescence due to auto-fluorescence. The background fluorescence is very similar to the fluorescence measured for the $B$. subtilis JH642 strain lacking gfpmut2. This implies that the $\mathrm{P}_{\text {spac }}$ promoter is tightly controlled.

Data acquisition and analysis. We collected cells from growth cultures at $\mathrm{OD}_{600} \approx 1.0$, which corresponds to the late exponential phase. To eliminate cell aggregates, we centrifuged cells at $4,000 \mathrm{rpm}$ for $1 \mathrm{~min}$, pelleted the supernatant at $14,000 \mathrm{rpm}$ for $1 \mathrm{~min}$ and resuspended the pellet in PBS. We independently confirmed the distributions of cell shapes using fluorescence microscopy. Single-cell fluorescence measurements were carried out on a Becton-Dickinson FACScan flow cytometer with a 488-nm Argon excitation laser and a 525-nm emission filter. FACScan data were analyzed on a Macintosh Quadra 650 using the Cell Quest program. During each flow-cytometer experiment, we collected data from $10^{4}-10^{5}$ single cells; each run typically lasted for $2 \mathrm{~min}$ and was conducted at room temperature. Cells from the same sample were often analyzed in two runs separated by $15 \mathrm{~min}$ or more. The measured fluorescence distribution was unchanged both during the course of a single run and between two such runs. To reduce noise in fluorescence values resulting from different cell sizes, we analyzed cells using the smallest allowed gate in the side-scattering and forward-scattering space.

Determination of transcriptional and translational efficiencies. For the translational mutants, we defined the transcriptional efficiency as the average fluorescence measured for a specific strain at a certain IPTG concentration normalized to the average fluorescence measured for that strain at full induction ([IPTG] $>1 \mathrm{mM}$ ). The translational efficiency of a strain was defined as the average fluorescence of the strain at full induction normalized to that of the wildtype strain (ERT25). For the transcriptional mutants, we defined transcriptional efficiency for each strain as the average fluorescence measured at full induction normalized to that of the ERT25 strain. We determined parameter error bars over at least 20 repeated measurements.

Modeling single gene expression. The noise properties of a single gene can be derived using the Langevin technique. This approach yields statistics equivalent to those generated by large-scale Monte Carlo simulations, but has the added advantage of providing insight into system behavior ${ }^{25}$. We treat the mRNA number $r$ and protein number $p$ as continuous quantities and assume that fluctuations are introduced by gaussian white noise sources:

$$
\frac{d r}{d t}+\gamma_{R} r=k_{R}+\eta_{R}, \quad \frac{d p}{d t}+\gamma_{P} p=k_{P} r+\eta_{P} .
$$

Here, $\gamma_{R}$ and $\gamma_{P}$ represent the decay rates of mRNA and protein, respectively; $k_{R}$ is the transcription rate and $k_{P}$ is the translation rate, so the rate of protein creation is $k_{P} r$ (Fig. $3 a$ ). $\eta_{R}$ and $\eta_{P}$ are white noise sources with the following statistics:

$$
\left\langle\eta_{i}(t)\right\rangle=0, \quad\left\langle\eta_{i}(t) \eta_{i}(\mathrm{t}+\tau)\right\rangle=q_{i} \delta(\tau), \quad i=R \text { or } P,
$$

where angular braces represent population averages, and $\delta$ is the Dirac $\delta$ function. The noise magnitudes $q_{i}$ are chosen so that they are consistent with the steady-state Poisson statistics of chemical reactions. For example, in steady-state, the mRNA number is given by $\langle r\rangle=k_{R} / \gamma_{R}$. Expanding around this steady-state by setting $r=\langle r\rangle+\delta r$ gives:

$$
\frac{d \delta r}{d t}+\gamma_{R} \delta r=\eta_{R}
$$

Fourier-transforming these equations by setting $x(t)=\int e^{i \omega t} x(\omega) d \omega / 2 \pi$ gives

$$
\frac{\delta r(\omega)}{\eta_{R}(\omega)}=\frac{1}{\gamma_{R}+i \omega}, \quad\left\langle\left|\eta_{R}(\omega)\right|^{2}\right\rangle=q_{R},
$$

so that the steady-state value of the fluctuations is given by

$$
\langle\delta r\rangle=\int \frac{d \omega}{2 \pi} \frac{1}{\gamma_{R}^{2}+\omega^{2}} q_{R}=\frac{q_{R}}{2 \gamma_{R}} .
$$

Now we impose Poisson statistics by setting $\left\langle\delta r^{2}\right\rangle=\langle r\rangle$, giving $q_{R}=2 k_{R}$, and similarly, $q_{p}=2 k_{P} k_{R} / \gamma_{R}$. Protein number fluctuations can then be derived as

$$
\left\langle\delta p^{2}\right\rangle=\int \frac{d \omega}{2 \pi} \frac{1}{\gamma_{P}^{2}+\omega^{2}}\left(q_{P}+\frac{k_{P}^{2} q_{R}}{\gamma_{R}^{2}+\omega^{2}}\right)=\langle p\rangle\left(1+\frac{k_{P}}{\gamma_{R}+\gamma_{P}}\right) .
$$

We define the noise strength to be the quantity $v=\left\langle\delta p^{2}\right\rangle /\langle p\rangle$, also known as the Fano factor (Fig. 1a). For a Poisson process, $v=1$; for an arbitrary stochastic process, the noise strength reveals deviations from Poissonian behavior. Setting $\phi=\gamma_{P} / \gamma_{R}$ and defining the burst size $b=k_{P} / \gamma_{R}$ finally gives

$$
\frac{\left\langle\delta p^{2}\right\rangle}{\langle p\rangle} \equiv \frac{\sigma_{p}^{2}}{\langle p\rangle}=1+\frac{b}{1+\phi} .
$$

Typically, $\phi$ is a small quantity (mRNA is unstable compared with protein), so that the result above reduces to that shown in the main text.

Monte Carlo simulations. Simulations were implemented using Gillespie's algorithm for stochastic coupled chemical reactions ${ }^{1}$. The reactions simulated are those schematically indicated in Fig. $3 a$. We assume individual reactions to be Poisson, so that the probability of a reaction with rate $k$ happening in a time $d t$ is given by $k d t$, and the waiting times between successive reactions are exponentially distributed. We assume that steady-state has been reached at a time equal to ten times the protein half-life. Each simulated histogram is the result of 5,000 trials.

Software. We converted data to ASCII format using MFI (E. Martz, Univ. of Massachusetts, Amherst, available at http://www.umass.edu/ $\mathrm{microbio} / \mathrm{mfi}$.

\section{Acknowledgments}

We thank S. Bell, E.M. Judd, H.H. McAdams, W.F. Burkholder and R. Weiss for critically reviewing the manuscript. E.O. was funded through a Merck fellowship. This work was supported by the Edgerly Science Partnership fund, DARPA and a National Science Foundation CAREER award.

\section{Competing interests statement}

The authors declare that they have no competing financial interests.

Received 9 November 2001; accepted 18 March 2002.

1. Gillespie, D.T. Exact stochastic simulation of coupled chemical reactions. J. Phys. Chem. 81, 2340-2361 (1977).

2. McAdams, H.H. \& Arkin, A. It's a noisy business! Genetic regulation at the nanomolar scale. Trends Genet. 15, 65-69 (1999).

3. McAdams, H.H. \& Arkin, A. Stochastic mechanisms in gene expression. Proc. Natl Acad. Sci. USA 94, 814-819 (1997).

4. Arkin, A., Ross, J. \& McAdams, H.H. Stochastic kinetic analysis of developmental pathway bifurcation in phage $\lambda$-infected Escherichia coli cells. Genetics 149, 1633-1648 (1998).

5. Elowitz, M.B. \& Leibler, S. A synthetic oscillatory network of transcriptional regulators. Nature 403, 335-338(2000)

6. Barkai, N. \& Leibler, S. Biological rhythms: circadian clocks limited by noise. Nature 403, 267-268 (2000).

7. Berg, O.G., Paulsson, J. \& Ehrenberg, M. Fluctuations and quality of control in biological cells: zero-order ultrasensitivity reinvestigated. Biophys. J. 79, 1228-1236 (2000). 
8. Thieffry, D., Huerta, A.M., Pérez-Rueda, E. \& Collado-Vides, J. From specific gene regulation to genomic networks: a global analysis of transcriptional regulation in Escherichia coli. BioEssays 20, 433-440 (1998).

9. Lutz, R. \& Bujard, H. Independent and tight regulation of the transcriptional units in Escherichia coli via the LacR/O, the TetR/O and $\mathrm{AraC} / \mathrm{I}_{1}-\mathrm{I}_{2}$ regulatory elements. Nucleic Acids Res. 25, 1203-1210 (1997).

10. LØbner-Olesen, A. Distribution of minichromosomes in individual Escherichia coli cells: implications for replication control. EMBO J. 18, 1712-1721 (1999).

11. Vellanoweth, R.L. \& Rabinowitz, J.C. The influence of ribosome-binding-site elements on translational efficiency in Bacillus subtilis and Escherichia coli in vivo. Mol. Microbiol. 6, 1105-1114 (1992).

12. Thattai, M. \& van Oudenaarden, A. Intrinsic noise in gene regulatory networks. Proc. Natl Acad. Sci. USA 98, 8614-8619 (2001).

13. Paulsson, J., Berg, O.G. \& Ehrenberg, M. Stochastic focusing: fluctuationenhanced sensitivity of intracellular regulation. Proc. Natl Acad. Sci. USA 97, 7148-7153 (2000)

14. von Dassow, G., Meir, E., Munro, E.M. \& Odell, G.M. The segment polarity network is a robust developmental module. Nature 406, 188-192 (2000).

15. Becskei, A. \& Serrano, L. Engineering stability in gene networks by autoregulation. Nature 405, 590-593 (2000)

16. Chapon, C. Expression of malT, the regulator gene of the maltose regulon in Escherichia coli, is limited both at transcription and translation. EMBO J. 1, 369-374 (1982).

17. Trotot, P. et al. Comparative analysis of the cya locus in enterobacteria and related Gram-negative facultative anaerobes. Biochimie 78, 277-287 (1996).

18. Botsford, J.L. \& Harman, J.G. Cyclic AMP in prokaryotes. Microbiol. Rev. 56, 100-122 (1992).
19. Spudich, J.L. \& Koshland Jr, D.E. Non-genetic individuality: chance in the single cell. Nature 262, 467-471 (1976).

20. Levi, M.D., Morton-Firth, C.J., Abouhamad, W.N., Bourret, R.B. \& Bray, D. Origins of individual swimming behavior in bacteria. Biophys. J. 74, 175-181 (1998)

21. Ptashne, M. et al. Autoregulation and function of a repressor in bacteriophage $\lambda$. Science 194, 156-161 (1976).

22. Weiss, R., Homsy, G.E. \& Knight Jr, T.F. Toward in vivo digital circuits. Proceedings of the DIMACS Workshop on Evolution as Computation (1999).

23. Hasty, J., McMillen, D., Isaacs, F. \& Collins, J.J. Computational studies of gene regulatory networks: in numero molecular biology. Nature Rev. Genet. 2, 268-279 (2001).

24. Gardner, T.S., Cantor, C.R. \& Collins, J.J. Construction of a genetic toggle switch in Escherichia coli. Nature 403, 339-342 (2000).

25. Kepler, T.B. \& Elston, T.C. Stochasticity in transcriptional regulation: origins, consequences and mathematical representations. Biophys. J. 81, 3116-3136 (2001).

26. Plumbridge, J. How to achieve constitutive expression of a gene within an inducible operon: the example of the nagC gene of Escherichia coli. J. Bacteriol. 178, 2629-2636 (1996).

27. Baumeister, R., Flache, P., Melefors, O., von Gabain, A. \& Hillen, W. Lack of a $5^{\prime}$ non-coding region in Tn1721 encoded tetR mRNA is associated with a low efficiency of translation and a short half-life in Escherichia coli. Nucleic Acids Res. 19, 4595-4600 (1991).

28. Kelley, R.L. \& Yanofsky, C. trp aporepressor production is controlled by autogenous regulation and inefficient translation. Proc. Natl Acad. Sci. USA 79, 3120-3124 (1982). 